\title{
Psychiatric Context of Acute/Early HIV Infection. The NIMH Multisite Acute HIV Infection Study: IV
}

\author{
J. Hampton Atkinson · Jenny A. Higgins · Ofilio Vigil · Robert Dubrow • \\ Robert H. Remien - Wayne T. Steward · Corinna Young Casey · \\ Kathleen J. Sikkema · Jackie Correale $\cdot$ Chris Ake $\cdot$ J. Allen McCutchan • \\ Peter R. Kerndt · Stephen F. Morin · Igor Grant
}

Published online: 11 June 2009

(c) The Author(s) 2009. This article is published with open access at Springerlink.com

\begin{abstract}
Acute/early HIV infection is a period of high risk for HIV transmission. Better understanding of behavioral aspects during this period could improve interventions to limit further transmission. Thirty-four participants with acute/early HIV infection from six US cities were assessed with the Mini International Diagnostic Interview, Beck Depression Inventory II, State-Trait Anxiety Inventory, Brief COPE, and an in-depth interview. Most had a preHIV history of alcohol or substance use disorder (85\%); a
\end{abstract}

J. H. Atkinson $(\bowtie) \cdot$ O. Vigil · C. Y. Casey · C. Ake .

J. A. McCutchan · I. Grant

HIV Neurobehavioral Research Center, University of California,

San Diego, 150 West Washington Street, La Jolla,

CA 92103, USA

e-mail: jhatkinson@ucsd.edu

J. A. Higgins

Center for Health and Wellbeing, Princeton University,

Princeton, NJ, USA

R. Dubrow

Center for Interdisciplinary Research on AIDS, Yale University, New Haven, CT, USA

R. H. Remien · J. Correale

HIV Center for Clinical and Behavioral Studies, New York State Psychiatric Institute and Columbia University, New York, NY, USA

W. T. Steward · S. F. Morin

Center for AIDS Prevention Studies, University of California,

San Francisco, CA, USA

K. J. Sikkema

Duke University, Durham, NC, USA

P. R. Kerndt

Sexually Transmitted Disease Program, Los Angeles County

Department of Public Health, Los Angeles, CA, USA majority (53\%) had a history of major depressive or bipolar disorder. However, post-diagnosis coping was predominantly adaptive, with only mild to moderate elevations of anxious or depressive mood. Respondents described challenges managing HIV in tandem with pre-existing substance abuse problems, depression, and anxiety. Integration into medical and community services was associated with adaptive coping. The psychiatric context of acute/early HIV infection may be a precursor to infection, but not necessarily a barrier to intervention to reduce forward transmission of HIV among persons newly infected.

Keywords Acute HIV infection - Psychiatric disorder . Substance use disorder . Coping

\section{Introduction}

Acute HIV infection (AHI) - the weeks to about 2 months between acquisition of HIV and completion of seroconversion-is characterized by extremely elevated concentrations of HIV RNA (viral load) in blood and genital secretions, which markedly increases risk of transmission. Furthermore, although acute HIV shedding is over about 10 weeks post-infection, elevated onward transmission likely extends through the period of early infection (defined as the 6 month period after seroconversion) due to ongoing high risk behaviors, associated sexually transmitted diseases that increase transmission risk, and transmission amplification through high-risk sexual and drug-use networks (for more detailed arguments and references, see [12], the first paper of this series in this issue of the journal). Efforts at secondary prevention aimed toward preventing forward transmission during acute/early infection may require not only an understanding of the infected 
individuals' awareness, behaviors, and knowledge of AHI, but also of the mental health context of infection itself.

Although the mental health of individuals with acute/ early infection has not been specifically studied, anxiety and depressive symptoms lasting for weeks or months after testing positive for HIV in chronically infected persons are well-documented (e.g., [16, 19, 20]). Most studies of HIVinfected persons in the developed world suggest there is an elevated lifetime and current prevalence of formal psychiatric and substance use disorders compared to the prevalence observed in population-based community surveys [4]. For example, up to $35-40 \%$ of HIV infected individuals have a lifetime history of major depression [1, $2,4]$, compared to around $15 \%$ of community controls [8], with the risk of major depression increasing in those with advanced medical disease [2, 16]. Similarly, lifetime prevalence of alcohol use disorders (i.e., abuse and dependence) and non-alcohol substance use disorders (e.g., methamphetamine, cocaine, opioids) in infected cohorts exceeds that in the community: 45-70\% [4] versus 18-30\% for alcohol use disorders [9] and $50 \%$ or more [4] versus $10 \%$ for non-alcohol substance use disorders [6]. Finally, the so-called "seriously mentally ill," defined as those with severe mood disorder (e.g., bipolar disorder) and psychoses are at heightened risk for acquiring HIV (e.g., [29]).

While these studies have documented the mental health status of people chronically infected with HIV, there is a dearth of knowledge about the mental health status of persons with acute/early infection. Study of such persons affords a unique glimpse into people's mental health immediately preceding and following HIV transmission and allows an exploration of their unique mental health needs.

This is the fourth in a series of five papers in this issue of the journal (see $[11,12,23,27]$ ) that describe results from the National Institute of Mental Health Multisite Acute HIV Infection Study (see [12] for the overall aims of the study). The present research evaluated coping approaches, current mood, and lifetime prevalence of mood and substance use disorders in a sample of people diagnosed with acute/early HIV infection. We believe that this is the first study of the psychiatric setting for and immediate responses to such infections.

\section{Methods}

Participants were 18 years of age or older, had sufficient English proficiency to complete the study measures, and had documented evidence of acute or early HIV infection (see [12]). Subjects were asked to participate in two study visits. The first visit was targeted to be held within 4 weeks of each participant being informed of his or her diagnosis, and a follow-up visit was targeted for 8 weeks later. At each visit, participants completed an in-depth qualitative interview and a structured quantitative survey (see [23]). The current study utilized quantitative data from the first visit, focusing on the mental health status and sexual behavior sections of the survey, and qualitative data from both visits.

\section{Measures}

The Mini International Neuropsychiatric Interview (MINI; Plus version 5.0; [25]), a semi-structured clinician-administered interview with documented reliability and validity, was used to ascertain suicidality and lifetime and recent occurrence of the seven DSM-IV psychiatric and substance use disorders: Major Depressive Disorder, Bipolar I and II Disorders, Alcohol Abuse/Dependence, and Substance Abuse/Dependence. The module on suicide inquires about recent (past month) suicidal ideation, its frequency and intensity, and about lifetime suicide plans and attempts. A "current suicide risk assessment" scale is used to derive a risk score (no, low, moderate, or high risk). Interviewers were trained to a criterion standard by investigators at the site responsible for this evaluation (University of California, San Diego). Audio recordings were periodically evaluated to ensure adherence to this standard. Interviewers at each site hand-scored the interview for diagnoses; the San Diego clinical team reviewed all protocols to confirm correct scoring procedures.

Participants completed the Beck Depression InventoryII (BDI-II; [3]) and the state scale of the State-Trait Anxiety Inventory (STAI, [26]). Total scores on the Beck II range from 0 to 63 , with higher scores indicating more depressed mood (i.e., scores from 0 to $13=$ minimal severity; $14-28=$ mild to moderate severity; 2963 = severely depressed mood). The time period assessed was the past 2 weeks. State STAI scores range from 20 to 80 , with higher scores indicating more anxiety. A mean score of 35.7 ( $\mathrm{SD}=10.4$ ) has been reported of working male adults, while mean scores of $47.7(\mathrm{SD}=13.2)$ and 42.4 (SD $=13.8$ ) have been reported for neuropsychiatric and general medical/surgical patients, respectively [26]. State anxiety was anchored to the present ("right now, right at this moment").

Participants rated their "recent" coping behaviors on the Brief COPE [5]. The measure yields two summary coping domain scores: adaptive coping (eight subscales: e.g., active coping, planning, positive reframing, acceptance) or less adaptive coping (six subscales: e.g., self-distraction, denial, substance use; [15]). Summary scores were generated by summing the subscale scores in each domain, normalized for the number of component subscales. A 
higher summary score indicates a higher level of adaptive or less adaptive coping.

We measured sexual behaviors using a structured survey (see [27]). To better understand participants' own articulations of their mental health, HIV diagnosis, and coping strategies, we utilized data from the qualitative interviews.

\section{Data Analysis}

We conducted descriptive analyses of lifetime and recent rates of psychiatric and substance use disorders and suicidality, as well as recent mood and coping. We used a nonparametric approach (Wilcoxon Rank Sums test) to assess the significance of differences in number of sexual partners across psychodiagnostic groups. We used paired $t$-tests to assess the difference between adaptive and less adaptive coping.

Qualitative interviews were coded for themes as described elsewhere in this series [23]. For the findings reported here, coding sorts and coding reports focused on the following themes: substance use before diagnosis; physical and mental health issues before diagnosis; coping and emotional response to diagnosis (including emotional/ psychological response to the news, sources of support, and future thoughts and orientation); behavioral response to the diagnosis (including substance use); and access of medical and/or mental health services.

\section{Results}

Structured Psychiatric and Psychological Assessment

The demographic characteristics of the 34 individuals (28 cases of AHI and 6 cases of early HIV infection) who were enrolled in the study are presented in a companion piece in this issue [12]. In brief, participants were predominantly Caucasian or Hispanic gay men in their late 20s or early 30s, who had at least some college education. Psychiatric history, as assessed by the M.I.N.I. Plus, is described in Table 1. The vast majority of participants (29 of 34; $85.3 \%$ ) met criteria for a lifetime history of an alcohol or other substance use disorder, with more than half (21 of 34; $61.8 \%$ ) meeting criteria for a recent (within the past 12 months) disorder. Furthermore, a majority of participants $(18$ of $34 ; 52.9 \%)$ met criteria for a lifetime diagnosis of mood disorder, either major depressive disorder or bipolar disorder. Co-morbidity - the lifetime occurrence of two of more conditions (e.g., alcohol use disorder and mood disorder)—was characteristic of the sample, with 18 participants $(52.9 \%)$ having two or more disorders.

As assessed with the M.I.N.I. Plus, a substantial proportion reported having made a suicide attempt at some
Table 1 Lifetime and recent prevalence of DSM-IV disorders in acute HIV infection $(N=34)$ compared to US lifetime estimates from population-based community surveys

\begin{tabular}{lcl}
\hline Disorder & $\begin{array}{l}\text { AHI cohort } \\
N=34\end{array}$ & $\begin{array}{l}\text { Community } \\
\text { lifetime \% }\end{array}$ \\
\hline Major depression (\%, No.) & $8.8(3)$ & $14.0^{\mathrm{a}}$ \\
Recent (last 2 weeks) lifetime & $29.4(10)$ & \\
Bipolar disorder (\%, No.) & $2.9(1)$ & $4.4^{\mathrm{a}}$ \\
Recent (current) lifetime & $23.5(8)$ & \\
Alcohol use disorder (\%) & $41.2(14)$ & $9.7^{\mathrm{a}} 36.7^{\mathrm{a}}$ \\
Recent (last 12 months) lifetime & $79.4(27)$ & \\
Other substance use disorder (\%) & $52.9(18)$ & $1.9^{\mathrm{a}}$ \\
Recent (last 12 months) lifetime & $64.7(22)$ & $14.3^{\mathrm{a}}$ \\
Current suicide ideation & $20.6(7)$ & $3.3^{\mathrm{b}}$ \\
Lifetime suicide attempt & $32.4(11)$ & $5^{\mathrm{c}}$ \\
\hline
\end{tabular}

Lifetime indicates: ever, recent or past occurrence

${ }^{a}$ National Epidemiologic Survey on Alcoholism and Related Conditions $[8,18,9,6]$

b National Comorbidity Survey Replication [14], last 12 months

c National Comorbidity Survey [13]

time in their life (11 of $34 ; 32.4 \%$ ). As might be expected, all individuals reporting prior suicide attempts also met lifetime criteria for alcohol and/or substance use disorders; likewise a lifetime diagnosis of mood disorder was prevalent in those with suicide attempts (7 of $11 ; 63.6 \%$ ). A significant minority of participants ( 7 of $34 ; 20.6 \%$ ) reported some form of suicidal ideation in the past month. This consisted of endorsements of thoughts about suicide, thoughts about being better off dead or wishing one were dead, and thinking of harming oneself. None reported a suicide attempt in the past month. Current suicide risk assessment scores ranged from 0 to 24 . The distribution of risk scores was as follows: no risk, $N=27$; low risk, $N=3$; moderate risk, $N=2$; high risk, $N=2$.

The relationship of mood and substance use disorders to sexual risk behavior was also assessed. The only finding that achieved even borderline statistical significance was that in the 2 months preceding documentation of HIV, those with a lifetime diagnosis of bipolar disorder $(N=8)$ reported more sexual partners than those with no lifetime mood disorder diagnosis (i.e., no major depression or bipolar disorder, $N=16) \quad(18.3 \pm 19.0$ partners vs. $4.4 \pm 4.5$ partners; $P=0.06)$.

In terms of the timing of onset of psychiatric condition, all participants with a lifetime diagnosis of mood or substance disorder reported onset preceded notification of HIV infection. The inventories of current mood revealed mild depressive (mean BDI-II $=12.0 \pm 8.9$ ) and anxiety (mean state scale of the STAI $=36.4 \pm 12.4$ ) symptoms. The vast majority of participants were experiencing minimal $(N=21 ; 61.8 \%)$ or mild-moderate $(N=11 ; 32.4 \%)$ 
symptoms of depressed mood, while only two (5.9\%) were experiencing severe depressive symptoms. Furthermore, participants reported greater use of adaptive coping approaches (summary Brief COPE adaptive domain score $=5.4 \pm 1.1$ ) compared to less-adaptive approaches to confronting their HIV diagnosis (summary Brief COPE less-adaptive domain score $=3.4 \pm 1.1$ ). This difference was highly significant $(P<0.0001)$.

\section{In-Depth Interview Findings}

Findings from the qualitative in-depth interviews corroborated those from the structured surveys. Respondents described the challenges of managing an HIV diagnosis in tandem with pre-existing substance abuse problems, depression, and anxiety. However, despite these challenges, several respondents appeared to be coping well, especially those well integrated into medical and social services and/or a community of other sero-positive individuals.

Although most respondents were coping well, in-depth interviews also revealed that psychiatric and substance disorders might have enhanced some individuals' vulnerability to infection and adversely impacted post-diagnosis coping. For example, one participant described pre-existing co-morbidity with bipolar disorder and addictive behavior. "I tend to overdo a lot of things," he said. "And I think I'm an addict with lots of different addictions: food, sex, et cetera." When recounting his infection story, he reported:

I remember fucking this black guy. I remember him sneezing and stuff, or coughing. I thought perhaps he might have been sick. And then, you know, [HIV] crossed my mind, but I'm like, 'Fuck it, I don't care.' I was pretty self-destructive. I still am pretty self destructive. (33-year old gay man, New York).

This respondents' post-diagnosis coping also was compromised by his experience with depression and suicidality:

I'd called many help lines, searching for help, searching for help, psychiatrically. When I was in my lows...thinking about going out and getting a gun. [..] But there's no help out there in New York City. So that's the reason a lot of people just go ahead and [commit suicide], and I totally understand that, totally.

Other respondents' infection and coping narratives were infused with substance abuse disorders, both as harbingers of infection and as factors in post-diagnosis coping. One participant described a long history of methamphetamine and cocaine use, which he factored into his HIV infection:
When you're high, you don't bother to ask anyone about HIV. [..] Do I think drugs have anything to do with me having [HIV]? Of course I do. (57-year old gay man from San Francisco).

Since diagnosis, this respondent had struggled to create a new, drug-free social network. Highlighting the cycle between mental illness and substance use, he also worried that anxiety about his diagnosis could lead him back to drug use. "I felt insecure enough without having HIV, he said. "Is this going to make me want to go back to past behavior with the drug use? It very easily could."

In a final example of this theme, one respondent described a history of both depression and methamphetamine use (he also reported extreme BDI-II severity on his structured interview). He expressed anxiety about how to have sex without methamphetamine and how to access the help he needed:

I'm trying to get the right help. But I'm not doing it right...I'm making the wrong choices, you know?

And I need help, but I can't-I'm-I'm alone with this. (31-year old gay man, Los Angeles).

In keeping with the structured interview findings, a number of respondents described highly functional coping in the aftermath of their HIV diagnosis, despite some of the mental health challenges mentioned above. Qualitative data indicated that two factors tended to be most strongly associated with adaptive coping: good access to clinical and social services; and integration into a community of sero-positive people and/or a close relationship with another HIV-positive person.

One respondent suspected that he became infected through one of his short-term Internet partners, but his long-term partner of 7 years was also HIV-positive. Because of this latter partner, he not only knew what to expect in the progression of the disease, but he received immediate referrals to both doctors and support services: "I've gotten a lot information on educational programs and stuff like that. With [my partner], these things have helped me get my focus back on reorganizing my life. [..] Until I talked to them, I didn't quite focus in on what the positive aspects of this could be (36-year old gay man, San Diego).

Similarly, another respondent seemed to be faring well after diagnosis, in large part because of the services available to him at his testing site, the Gay and Lesbian Center in Hollywood:

They offered me medical assistance, psychological assistance, financial assistance, um, moral assistance. [..] Being newly infected, I'm...I'm eligible for a whole bunch of stuff. You know, house....assistance in housing, assistance in medical care, and stuff like 
this. [..] I'm constantly amazed at the medical staff. (46-year old gay man, Los Angeles).

Perhaps due to this support, the above respondent had not used methamphetamine since diagnosis, and he was optimistic about his ability to stay clean.

\section{Discussion}

This study suggests the psychiatric context of acute/early infection encompasses an elevated prevalence of psychiatric disorders exceeding those observed in recent national community-based epidemiologic surveys $[6,8,9,18]$ combined with current anxious and depressive symptoms which in the main were not so severe as to interfere with efforts at early intervention. Furthermore, we found that with the right resources, individuals with acute/early HIV infection reported highly adaptive coping strategies.

Our sample's rates of mood, substance use disorders, and psychiatric co-morbidity, with onset pre-HIV infection, were consistent with studies of "chronically" infected individuals $[2,4]$. One in five of study participants had a bipolar disorder, consistent with evidence that persons with this disorder may be at heightened risk for HIV [24, 29].

Current mood symptoms of depression and anxiety were mildly elevated, although within the range of previous studies of individuals seen at voluntary testing centers after notification of seropositivity in the era before modern antiretroviral treatments [21]. However, almost $40 \%$ of our participants did have depression symptom scores in the "clinical" range (BDI-II > 13). In the earlier research of chronically infected individuals studied at the point of notification, those in the clinical range at initial testing were likely to have clinically significant symptoms at 1 year follow-up [21].

Suicidality in the context of HIV/AIDS has long been a concern among the medical and general community [17]. The current prevalence of suicidal ideation in our study of $21 \%$ and the lifetime prevalence of suicide attempts of $32 \%$ exceeded the community lifetime prevalence of suicidal ideation $(14 \%,[13,14])$ and attempts (5\%, [13]). There were no reports of suicide attempts among our participants in the immediate aftermath of testing positive. Those previously attempting suicide typically had lifetime histories of mood or substance use disorders, which are known to elevate risk of attempts. In two individuals (5.9\%) rated at "high risk" for suicide, it was unclear whether suicidality antedated or resulted from testing positive. These results are consistent with the notion that most suicidal behavior in HIV-infected persons is related to mood or substance use disorder rather than HIV.
Our findings suggest implications for development of interventions for primary or secondary HIV prevention. The mild current mood symptoms, lack of acute suicidal behavior, and predominant reliance on adaptive coping approaches indicate that a substantial proportion of individuals diagnosed with acute/early HIV infection remain generally hopeful and forward-looking despite the stress of notification of positive testing, suggesting that appropriate prevention interventions would have a high probability of success. Behavioral interventions for people living with HIV might be adapted to apply to the period of acute/early infection [7, 10, 28]. However, ongoing intervention may be needed for persons with conditions associated with episodic difficulties with impulse control (e.g., bipolar disorder), since reductions in risky sexual behavior after testing positive may be difficult to sustain. With regard to primary prevention, individuals with mood disorders may be especially vulnerable, given the nature of the depressive illness and the likelihood of co-existing substance use disorders. Therefore public mental health centers might particularly be brought into the network of sites promoting testing and education about AHI.

There are several important limitations to this research that may impact on its generalizability. First, our sample size was very small. Next, we only included individuals who were diagnosed in the stage of acute/early infection, whereas most infected persons are diagnosed at a later stage [22]. It is possible that persons diagnosed with $\mathrm{AHI}$ in particular have specific characteristics that make them more likely to be identified and more likely to have the mental health characteristics observed in our sample. The small sample makes our estimates of the prevalence of psychiatric disorders imprecise; for bipolar disorder, for example, the difference of a few cases would markedly alter our proportions. It may also be the case that by recruiting from sites offering testing and treatment we sampled for individuals already involved in other systems of care, including mental health or substance abuse care systems. Recruiting a population with a high base rate of psychopathology would overestimate rates of mood and substance use disorders in persons with acute/early HIV infection. Another limitation is that we did not determine whether people diagnosed with acute/early infection experience greater distress than chronically infected individuals. Knowing if episodes of mental disorder or distress tends to be more likely during the time surrounding acute/ early infection would help inform interventions. Finally, our sample consisted of predominantly gay men from major urban areas with well-organized gay communities. This may account for the high level of adaptive coping observed in our sample, with the concomitant access to clinical and social services and integration into an HIVpositive "community." 
Despite these limitations it is possible that including mental health considerations into behavioral prevention perspectives may help expand and advance development of interventions aimed at reducing risk of HIV transmission and acquisition.

Acknowledgments Primary funding for this study was provided by the National Institute of Mental Health as supplements to the following AIDS Research Centers: P30MH062246, Center for AIDS Prevention Studies, University of California San Francisco; P30MH043520, HIV Center for Clinical and Behavioral Research, New York State Psychiatric Institute and Columbia University; P30MH062512, HIV Neurobehavioral Research Center, University of California San Diego; P30MH052776, Center for AIDS Intervention Research, Medical College of Wisconsin; P30MH058107, Center for HIV Identification, Prevention and Treatment Services, University of California Los Angeles; and P30MH062294, Center for Interdisciplinary Research on AIDS, Yale University. Additional funding was provided by: P30AI42853, Lifespan/Tufts/Brown Center for AIDS Research and AI43638, Acute Infection and Early Disease Research Program, University of California San Diego. Complete details about funding, the study Steering Committee, co-investigators, collaborating scientists, and project staff are presented in the first paper of this series [12].

Open Access This article is distributed under the terms of the Creative Commons Attribution Noncommercial License which permits any noncommercial use, distribution, and reproduction in any medium, provided the original author(s) and source are credited.

\section{References}

1. Atkinson JH, Grant I, Kennedy CJ, Richman DD, Spector SA, McCutchan JA. Prevalence of psychiatric disorders among men infected with human immunodeficiency virus: a controlled study. Arch Gen Psychiatry. 1988;45:859-64.

2. Atkinson JH, Heaton RK, Patterson TL, Wolfson T, Deutsch R, Brown SJ, et al. Two-year prospective study of major depressive disorder in HIV-infected men. J Affect Disord. 2008;108(3):22534.

3. Beck A, Steer R, Brown G. BDI-II Manual. San Antonio, TX: The Psychological Corporation; 1996.

4. Bing EG, Burnham MA, Longshort D, Fleishman JA, Sherbourne $\mathrm{CD}$, London AS, et al. Psychiatric disorders and drug use among human immunodeficiency virus-infected adults in the United States. Arch Gen Psychiatry. 2001;58:721-8.

5. Carver CS. You want to measure coping but your protocol is too long: consider the Brief COPE. Int J Behav Med. 1997;4:92-100.

6. Compton WM, Thomas YF, Stinson FS, Grant BF. Prevalence, correlates, and comorbidity of DSM-IV drug abuse and dependence in the United States. Arch Gen Psychiatry. 2007;64:566-76.

7. Crepaz N, Lyles C, Wolitski RJ, Passin W, Rama S, Herbst JH, et al. Do prevention interventions reduce HIV risk behaviors among people living with HIV? A meta-analytic review of controlled trials. AIDS. 2006;20:143-57.

8. Hasin DS, Goodwin RD, Stinson FS, Grant BF. Epidemiology of major depressive disorder. Arch Gen Psychiatry. 2005;62:1097106.

9. Hasin DS, Stinson FS, Ogburn E, Grant BF. Prevalence, correlates, disability, and comorbidity of DSM-IV alcohol abuse and dependence in the United States. Arch Gen Psychiatry. 2007;64:830-42.

10. Johnson BT, Carey MP, Chaudoir SR, Reid AE. Sexual risk reduction for persons Living with HIV: research synthesis of randomized controlled trials, 1993-2004. J Acquir Immune Defic Syndr. 2006;41:642-50.

11. Kelly JA, Morin SF, Remien RH, Steward WT, Higgins JA, Seal DW, et al. Lessons learned about behavioral science and Acute/ Early HIV infection. The NIMH multisite Acute HIV infection study: V. AIDS Behav. (this issue) (2009).

12. Kerndt PR, Dubrow R, Aynalem G, Mayer KH, Beckwith C, Remien RH, et al. Strategies used in the detection of Acute/Early HIV infections. The NIMH multisite Acute HIV infection study: I. AIDS Behav. (this issue) (2009).

13. Kessler RC, Borges G, Walters EE. Prevalence of and risk factors for lifetime suicide attempts in the National Comorbidity Survey. Arch Gen Psychiatry. 1999;5:617-26.

14. Kessler RC, Berglund P, Borges G, Nock M, Wang PS. Trends in suicide ideation, plans, gestures, and attempts in the United States, 1990-1992 to 2001-2003. J Am Med Assoc. 2005;293:2487-95.

15. Lunsford SL, Simpson KS, Chavin KD, Hildebrand LG, Miles LG, Shilling LM, et al. Racial differences in coping with the need for kidney transplantation and unwillingness to ask for live organ donation. Am J Kidney Dis. 2006;47:324-31.

16. Lyketsos CG, Hoover DR, Guccione M, Dew MA, Wesch JE, Bing EG, et al. Changes in depressive symptoms as AIDS develops. Am J Psychiatry. 1996;153:1430-7.

17. Marzuk PM, Tierney H, Tardiff K, Gross EM, Morgan EB, Hsu MA, et al. Increased risk of suicide in persons with AIDS. J Am Med Assoc. 1988;259(9):1333-7.

18. Merikangas DR, Akiskal HS, Angst J, Greenberg PE, Hirschfield RMA, Petukhova M, et al. Lifetime and 12-month prevalence of bipolar spectrum disorder in the National Comorbidity Survey Replication. Arch Gen Psychiatry. 2007;64:543-52.

19. Morin SF, Charles KA, Malyon AK. The psychological impact of AIDS on gay men. Am Psychol. 1984;39:1288-93.

20. Perry S, Fishman B, Jacobsberg L, Young J, Frances A. Effectiveness of psychoeducational interventions in reducing emotional distress after human immunodeficiency virus antibody testing. Arch Gen Psychiatry. 1991;48(2):143-7.

21. Perry S, Jacobsberg LR, Card CAL, Ashman T, Frances A, Fishman B. Severity of psychiatric symptoms after HIV testing. Am J Psychiatry. 1993;150:775-9.

22. Pilcher CD, Fiscus SA, Nguyen TQ, Foust E, Wolf L, Williams D, et al. Detection of acute infections during HIV testing in North Carolina. N Engl J Med. 2005;352:1873-83.

23. Remien RH, Higgins JA, Correale J, Bauermeister J, Dubrow R, Bradley M, et al. Lack of understanding of Acute HIV infection among newly-infected persons-implications for prevention and public health. The NIMH multisite Acute HIV infection study: II. AIDS Behav. (this issue) (2009).

24. Rosenberg SD, Goodman LA, Osher FC, Swartz MS, Essock SM, Butterfield MI, et al. Prevalence of HIV, hepatitis B, and hepatitis $\mathrm{C}$ in people with severe mental illness. Am J Public Health. 2001;91:31-7.

25. Sheehan DV, Lecrubier Y, Harnett-Sheehan K, Amorim P, Janavs J, Weiller E, et al. The Mini International Neuropsychiatric Interview (M.I.N.I.): the development and validation of a structured diagnostic psychiatric interview. J Clin Psychiatry. 1998;59(suppl 20):22-33.

26. Spielberger CD, Gorsuch RL, Lushene RE. STAI manual for the state-trait anxiety inventory. California: Consulting Psychologists Press; 1974.

27. Steward WT, Remien RH, Higgins JA, Dubrow R, Pinkerton SD, Sikkema et al. Behavior change following diagnosis with Acute/ 
Early HIV infection-a move to serosorting with other HIVinfected individuals. The NIMH multisite Acute HIV infection study: III. AIDS Behav. (this issue) (2009).

28. The NIMH Healthy Living Project Team. Effects of a behavioral intervention to reduce risk of transmission among people living with HIV: the healthy living project randomized controlled study. J Acquir Immune Defic Syndr. 2007;44:213-21.

29. Walkup J, Crystal S, Sambamoorthi U. Schizophrenia and major affective disorder among Medicaid recipients with HIV/AIDS in New Jersey. Am J Public Health. 1999;89:1101-3. 